\title{
EL SEPTENTRIÓN FLUVIAL Y MARÍTIMO DE MÉXICO EN EL DERECHO INTERNACIONAL GENERAL
}

\section{Juan de Dios GUTIÉRREZ BAYLÓN*}

RESUMEN: En el presente artículo se examina la crisis de los regímenes convencionales que México y Estados Unidos de América han suscrito en el último siglo en materia de ríos y fronteras marítimas. La tesis central de estudio es que los acuerdos bilaterales en materia de aprovechamiento hidrológico en la cuenca de los ríos fronterizos no se encuentran acordes con el derecho internacional general. El autor vincula los desarrollos del derecho internacional en materia de desertización, cambio climático y protección del medio ambiente, con las reglas que estima obsoletas, derivadas del tratado sobre ríos internacionales con la unión americana. Un esfuerzo análogo emprende el profesor Gutiérrez Baylón al analizar el acuerdo por el cual ambos países delimitan equivocadamente la plataforma continental en la zona del Golfo de México, en donde el derecho internacional general debe servir como elemento corrector.

ABSTRACT: This article examines the different river and maritime boundary conventions executed between Mexico and the United States of America during the last century. The author's main thesis is that the bilateral treaties regarding hydrologic exploitation of the frontier basin, are not in accordance with general international law. The author discusses the development of international law on blight, climatic change and environmental protection, in light of what he considers to be the obsolete rules derived from the Treaty on international rivers executed with the United States. A similar effort is carried out by professor Gutierrez Baylon, when he analyzes the convention whereby both countries inaccurately delimit the continental shelf of the Mexican Gulf. The author argues that general international law must be used to correct this problem.

RÉSUMÉ: Cet article fait l'examen de la crise du régime conventionnel entre le Mexique et les États-unis dans les domaines des fleuves et frontières maritimes. La thèse principal de ce travail est dans le sens que les traités bilateraux concernant l'exploitation hydrologique dans le bassin frontalier n'est suive pas les principes du droit international général. L'auteur fait la liason entre le développement du droit international dans le domain de désertification, changement climatique et protection de l'environement avec les règles dépassés du Traité sur les fleuves internationaux avec l'Union americaine. M. Gutiérrez Baylón fait la même démarche en ce qui concerne l'analyse de la convention conclue entre les deux pays pour aboutir à la délimitation du plateau continental dans la région du Golfe du Mexique, vis-à-vis du droit international général, dont celui-ci joue une fonction correctrice.

* Profesor de derecho internacional de la División de Estudios de Posgrado de la Facultad de Derecho de la UNAM. 
SUMARIO: I. El tratado entre México y Estados Unidos de América sobre ríos internacionales y la desertización regional. II. El régimen fluvial entre México y Estados Unidos de América, y el cambio fundamental de circunstancias en el derecho internacional. III. El Polígono Occidental en el Golfo de México y el

Timor Gap. IV. Conclusiones. V. Bibliografía.

\section{EL TRATADO ENTRE MÉXICO Y ESTADOS UNIDOS \\ DE AMÉRICA SOBRE RÍOS INTERNACIONALES \\ Y LA DESERTIZACIÓN REGIONAL}

El Tratado relativo al Aprovechamiento de las Aguas de los Ríos Colorado y Tijuana, y del Río Bravo (Grande) desde Fort Quitman, Texas, hasta el Golfo de México, ${ }^{1}$ (en lo sucesivo: Tratado bilateral sobre ríos internacionales), fue un tratado favorecido por el entorno diplomático bilateral que la Segunda Guerra Mundial dejó entre México y Estados Unidos de América. ${ }^{2}$ La puesta en marcha de la presa de irrigación Hoover y la apremiante necesidad de agua del estado de Texas incidieron en la urgencia bilateral por firmar una acuerdo en la materia. En algún sentido el gobierno mexicano tuvo la habilidad para servirse de las disputas interestaduales de algunas entidades federativas estadounidenses en materia de gestión hidrológica, al tiempo de que logró un buen acuerdo en lo que concierne a volúmenes de agua, en detrimento a la calidad y tratamiento de éstas. En este punto se dio inicio a una gran cantidad de diferendos (jamás debidamente atendidos sobre salinización y contaminación de los ríos internacionales de la región en los años subsecuentes). Una de las utilidades técnicas del acuerdo consistía en la posibilidad de que ambos Estados irrigaran regiones de sus territorios con aguas provenientes del extranjero, irrigación ésta que sería exponencialmente costosa si ambas partes pretendiesen hacerlo, sirviéndose de manera exclusiva de las avenidas naturales existentes en los espacios bajo sus respectivas soberanías.

1 Cfr. Diario Oficial de la Federación, 30 de marzo de 1946. Firmado en Washington, D. C., el 3 de febrero de 1944. El 14 de noviembre de 1944 se firmó en esa misma ciudad un protocolo adicional. El canje de los instrumentos se realizó el 8 de noviembre de 1945.

2 Para un análisis del entorno diplomático de la negociación del Tratado bilateral sobre ríos internacionales, cfr. Zorrilla, Luis G., Historia de las relaciones entre México y Estados Unidos de América, México, Porrúa, 1977, t. II, pp. 512 y ss.; Torres, Blanca, México y el mundo. Historia de sus relaciones exteriores, t. VIII, p. 51; Zoraida Vázquez, Josefina y Meyer, Lorenzo, México frente a Estados Unidos, México, Fondo de Cultura Económica, 1994, p. 190. 
Para el derecho internacional fluvial de la época, la regla sobre extracción, desfogue y desvío de recursos hidráulicos de ríos internacionales en zonas no limítrofes, derivaba si acaso del ámbito puramente convencional. $^{3}$

En rigor la crisis hidrológica regional en el septentrión mexicano tiene mucho que ver con la mutilación territorial decimonónica del Estado nacional, pues los mecanismos de irrigación heredados por México suponían la unidad jurídico administrativa de la entidades involucradas en la gestión de las aguas. ${ }^{4} \mathrm{La}$ involución de problema siguió históricamente conociendo arreglos, ${ }^{5}$ entendimientos ${ }^{6}$ y parches técnicos, trasunto de una cooperación verticalizada y temporal entre ambas naciones. No se entiende la historia diplomática del Estado nacional mexicano si no se comprende su lucha internacional por el agua en el norte del país.

Entre los éxitos de los mexicanos estuvo negociar en un sólo tratado los tres ríos, dándole así un tratamiento integral al problema fluvial bilateral. En el entramado fluvial fronterizo, las proporciones de los ríos es interesante. Respecto del río Bravo la aportación hidrológica de su cuenca es bastante equilibrada entre los dos países correspondiendo a un $47.9 \%$ para México y $52.1 \%$ para Estados Unidos de América. Por lo que toca al

3 Cfr. Brownlie, Ian, Principles of Public International Law, Oxford, Fifth Edition, 1998, p. 268; Nguyen, Quoch Dinh et al., Droit international public, LGDJ, 1994, p. 1143. Quizás analógicamente se podría sostener que ya en la época de firma del Tratado bilateral sobre ríos internacionales ya se visualizaba una nueva regla de instauración de un régimen internacional de aguas: "The notion of an international strait is always connected with a minimum of special utility, sufficent to justify the restriction of the rights of the coastal State...", The Corfu Channel, Judgment, Dissenting Opinon by Judge Azevedo, ICJ Reports 1949, p. 106.

$4 C$ fr. Margadant S., Guillermo F., "El régimen jurídico de las aguas no marítimas, en la literatura iusromanista desde los posglosadores hasta mediados del siglo XVIII", Boletín Mexicano de Derecho Comparado, México, UNAM, núm. 57, 1986. Véase, en particular, la nota de la página 55. De hecho, don Toribio Esquivel Obregón llegó documentadamente a afirmar que todas las aguas objeto del tratado eran de propiedad nacional tanto por los derechos históricos sobre ellas, como por aplicación de principios generales de derecho extrapolados al derecho internacional, en donde el territorio mexicano fungía como predio superior, así como por la prescripción adquisitiva sobre las mismas. "Impugnación del Tratado de Aguas", Revista de la Escuela Nacional de Jurisprudencia, México, UNAM, t. VIII, núm. 30, 1946, p. 206.

5 Entre éstos destaca la Convención para el Establecimiento de una Comisión Internacional que decida las Cuestiones de Límites que se susciten en el Cauce de los Ríos Bravo del Norte y Colorado, firmado en la Ciudad de Washington el 1 de marzo de 1889. La función de este tratado era a su vez, de acuerdo con su preámbulo: “...facilitar la ejecución de los principios contenidos en el Tratado de 12 de noviembre de 1884...". Posteriormente, mediante el tratado del 21 de mayo de 1906 se institucionalizó el régimen internacional de entregas de aguas entre los Estados.

6 La Comisión Internacional de Límites y Aguas entre México y Estados Unidos de América ha realizado una constante labor legiferante a través de las llamadas "actas" que rebasan sobradamente el número de trescientas. 
enorme río Colorado, la parte mexicana es prácticamente insignificante correspondiéndole a México sólo el $0.72 \%$. El río Tijuana tiene una cuenca de $72 . \%$ de aguas para México y $27.60 \%$ para Estados Unidos de América, sus volúmenes acuíferos no son detallados por el tratado. ${ }^{7}$

El grave problema hidrológico de México tiene también causalmente muchos factores de carácter endógeno derivados de cuestiones de impacto ambiental, vinculados la mayor parte de ellos a problemas propios del subdesarrollo como los asentamientos humanos no controlados, el desorden en la producción rural o incluso de retraso administrativo en la gestión y producción petrolera, eléctrica o nuclear. Empero, para los fines del derecho internacional del medio ambiente la dependencia económica o la insuficiencia tecnológica de un Estado son tan sólo elementos que explican el fenómeno multifactorial de la degradación hidrológica. ${ }^{8}$

Por una malformación sistémica en el derecho internacional general, el derecho fluvial internacional parece desarrollarse regionalmente al margen de las grandes controversias doctrinarias del derecho de gentes. ${ }^{9}$ Ciertamente desde el punto de vista histórico los Estados fluvialmente fronterizos suelen tener problemas de navegación, contaminación y aprovechamiento hidroeléctrico, dejando en un lugar casi secundario los problemas de irrigación, que en casos como el de México y Estados Unidos

7 Estos cálculos corresponden a la época en que se realizó el tratado vigente. $C f r$. Zorrilla, Luis G., op. cit., nota 2, pp. 512 y 513. Es importante la lectura de los ya célebres artículos 4o. (relativo al río Bravo) y 10 (relativo al río Colorado) del Tratado bilateral sobre ríos internacionales, en donde se establece el régimen de distribución hidráulica, así como la cláusula del caso fortuito. Dicha cláusula está llamada a ser histórica en el derecho internacional regional: "En caso de extraordinaria sequía o de serio accidente en los sistemas hidráulicos de los afluentes mexicanos aforados que hagan difícil para México dejar escurrir los 431721000 metros cúbicos (350 000 acres pies) anuales que se asignan a Estados Unidos de América como aportación mínima de los citados afluentes mexicanos, en el inciso c) del párrafo B de este artículo [se refiere a la afluencia de aguas provenientes de los ríos Conchos, San Diego, San Rodrigo, Escondido, Salado y Arroyo de las Vacas] los faltantes que existieren al final del ciclo aludido de cinco años, se repondrán en el ciclo siguiente con agua procedente de los mismo tributarios. "Siempre que la capacidad útil asignada a los Estados Unidos de por lo menos dos de las presas internacionales principales, incluyendo la localizada más aguas arriba, se llene con aguas pertenecientes a los Estados Unidos, se considerará terminado un ciclo de cinco años y todos los débitos totalmente pagados, iniciándose, a partir de ese momento, un nuevo ciclo". Cfr. Enríquez Coyro, Ernesto, El Tratado entre México y Estados Unidos de América sobre Ríos Internacionales, México, UNAM, 1976, t. II, pp. 885 y siguientes.

8 En sentido similar, las conclusiones del profesor Kiss, Alexandre, Droit international de l'environnement, París, Pedone, 1989, pp. 36 y 37.

9 La literatura jurídica de cuño anglosajón ha tocado la banalidad ante un problema grave y urgente que involucra la supervivencia de grandes cantidades de seres humanos. Cfr. Benvenisti, Eyal, "Collective Action in the Utilization of Shared Freshwater: The Challenges of International Water Resources Law”, American Journal of International Law, vol. 90, núm. 3, julio de 1996. 
de América se ha abandonado a la mera relación de fuerzas entre ambas naciones. Por un problema de ignorancia científica, subdesarrollo tecnológico o inestabilidad diplomática, más de medio siglo después de la firma y entrada en vigor del Tratado bilateral sobre ríos internacionales, se pretende seguir viendo el problema de la desertización regional, la insuficiencia hidráulica y retardo hidrológico como un asunto puramente bilateral, desdeñando así los mecanismos universales de prevención y rehabilitación ambiental. A finales de la Segunda Guerra Mundial quizás podría aceptarse que los volúmenes de irrigación de los ríos podrían aislarse del resto de factores naturales y antropogénicos del entorno pero ningún enfoque epistemológico estricto podría suscribir una proposición de esta naturaleza en la actualidad.

El sistema jurídico fluvial internacional está en búsqueda de nuevos regímenes de explotación y conservación sustentable. ${ }^{10} \mathrm{El}$ criterio "fundamentalista" de atribución en el derecho del mar, i. e. en el derecho hidrológico internacional, ya está superado por el derecho internacional general. ${ }^{11}$ En el momento actual se puede anunciar la formación de una regla de derecho internacional general que se sobrepone decididamente a las fórmulas convencionales bilaterales o regionales de uso voluntarista de las aguas dulces internacionales. Una justicia distributiva con criterios de sustentabilidad como elementos correctores subyace en el actual derecho fluvial internacional. ${ }^{12}$ Se ha llegado a enunciar una doctrina sobre principios equitativos sobre repartición de los volúmenes de aprovechamiento entre Estados fronterizos por ríos, lo que sin duda guarda algún grado de parentesco con la doctrina análoga en la delimitación marítima internacional. $^{13}$

Sobre los problemas periféricos en la gestión hidrológica internacional siempre ha sido difícil enunciar reglas generales de derecho, ${ }^{14}$ y su codificación se caracteriza por ser señaladamente lenta. Ciertamente el valor jurídico de los trabajos de la Comisión de Derecho Internacional ${ }^{15}$

10 Cfr. Jutta Brunnée, Stephen J. Toope, "The Changing Nile Basin Regime: Does Law Matter?", Harvard International Law Journal, vol. 43, núm. I, 2002, pp. 158 y 159.

11 Cfr. North Sea Continental Shelf, Judgment, ICJ Reports 1969, p. 30.

12 Cfr. Gabcíkovo-Nagymaros Project (Hungary/Slovakia), Judgment, ICJ Reports 1997, p. 78.

13 Cfr. International Law of Water Resources, Slavko Bogdanovic (coord.), Kluwer Law International, The Netherlands, 2001, pp. 252 y 412.

$14 C f r$. Fisheries case, (United Kingdom v. Norway), Judgment of December 18 th, 1951, ICJ Reports 1951, pp. 127, 131 y 132.

15 Convention sur le droit relatif aux utilisations des cours d'eau internationaux à des fins autres que la navigation, Rapport de la Sixième Commission réunie en Groupe de travail plénier, Président 
no rebasa hasta el momento actual el carácter de lex ferenda dentro del conjunto de reglas de derecho internacional aplicable al manejo de las aguas internacionales de los dos países. No obstante ello, no se justifica de manera alguna que los referidos Estados no hayan pretendido ni por asomo, buscar en los esfuerzos codificadores y de desarrollo de la mencionada comisión, buscar los mecanismos técnicos de solución, ni hacer entroncar el vetusto tratado sobre ríos internacionales a las condiciones del derecho internacional contemporáneo.

Si bien es cierto que la internacionalización de un río tiene como origen un acto bilateral, dicha internacionalización es tal en sentido estricto de la misma manera como lo son los canales y estrechos internacionales, con la condición de que los Estados ribereños tienen el privilegio de establecer consensuadamente las mejores condiciones de su explotación racionalizada en beneficio de las poblaciones de la región. ${ }^{16}$ La interdependencia de los Estados en materia ambiental es un principio de derecho internacional general (de origen interamericano), de donde deriva la obligación creciente de tomar como azimuth de la regla de derecho la estabilidad del recurso natural que sirve de sustento a la región. ${ }^{17}$ Dado el régimen internacional así establecido la habilitación a la comunidad internacional para su protección en aplicación del derecho internacional general es ineluctable. ${ }^{18}$

M. Chusei Yamada, A/51/869, 1997 (en lo sucesivo: Convención Internacional sobre la utilización del agua).

16 "According to international law, in order for a measure to be characterized as a 'conservation a management measure', it is sufficient that its purpose is to conserve and manage living resources and that, to this end, it satisfies various technical requirements". Fisheries Jurisdiction (Spain $v$. Canada), Jurisdiction of the Court, Judgment, ICJ Reports 1998, p. 461.

17 En el caso de Golfo de Fonseca, se sostuvo: "Invocar los atributos de la soberanía para realizar actos que puedan deparar daño o peligro a otro país, es desconocer el principio de la interdependencia de los Estados que les obliga a respetarse mutuamente y a abstenerse de todo aquello que involucre lesión, aunque sea potencial, para los derechos fundamentales de las demás entidades internacionales, que a semejanza de los individuos, tienen el derecho de vivir y desarrollarse sin detrimento una de otra, y si estos principios son de naturaleza indeclinable en la vida internacional, revisten mayor prestigio tratándose de los países de Centro América que en algunas ocasiones han incorporado tales postulados al rango de principios básicos de su derecho público". Corte de Justicia Centroamericana, Sentencia, Párrafo III, 9 de marzo de 1917. "The Tribunal, therefore, finds that the above decisions, taken as a whole, constitute an adequate basis for its conclusion, namely, that, under the principles of international law, as well as of the law of the United States, no State has the right to use or permit the use of its territory in such a manner as to cause injury by fumes in or to the territory of another or the properties of persons therein, when the case is of serious consequence and the injury is established by clear and convincing evidence". The Trail Smelter case (United States of America $v$. Canada), Award of March 11, 1941, Reports of International Arbitral Awards, United Nations, vol. III, p. 1965.

18 "The Court recognizes that the environment is under daily threat and that the use of nuclear 
Los contenciosos con incidencia específicamente en la materia fluvial ante la Corte Internacional de Justicia (CIJ) son crecientes creando un derecho jurisprudencial en la materia, ${ }^{19}$ y estableciendo un sistema de definiciones sobre temas en la materia que ya se estimaban claramente concebidos. ${ }^{20}$ Es también de inferirse que el suministro internacional de aguas potables está tocando la base de la teoría general de los derechos humanos. ${ }^{21}$ Atento a lo anterior, los acuerdos recurrentes de carácter paliativo entre México y Estados Unidos de América en materia de reconducción fluvial, que no prevén una profilaxis de conformidad con las reglas universales en materia ambiental, en los rubros hidrológico, climático o de biodiversidad, son violatorios del derecho internacional general en tanto que contribuyen al deterioro ecológico planetario. ${ }^{22}$

Por otro lado, la insuficiencia del derecho subregional de América del Norte ha quedado manifestada en la incapacidad de los órganos de solución de diferendos para invocar de manera cabal los criterios de desarrollo del derecho internacional ambiental, más allá de los factores puramente

weapons could constitute a catastrophe for the environment. The Court also recognizes that the environment is not an abstraction but represents the living space, the quality of life and the very health of human beings, including generations unborn. The existence of the general obligation of States to ensure that activities within their jurisdiction and control respect the environment of other States or of areas beyond national control is now part of the corpus of international law relating to the environment." Legality of the Threat or Use of Nuclear Weapons, Advisory Opinion, ICJ Reports 1996, pp. 241 y 242.

$19 C f r$. Land, Island and Maritime Frontier Dispute (El Salvador/Honduras: Nicaragua interveining), Judgment of 11 September 1992, ICJ Reports 1992, p. 547.

20 "The Court notes that various definitions of the term 'thalweg' are found in treaties delimiting boundaries and that the concepts of the thalweg of a watercourse and the centre of a watercourse are not equivalent. The word 'thalweg' has variously been taken to mean 'the most suitable channel for navigation' on the river 'determined by the line of deepest soundings', or 'the median line of the main channel followed by boatmen travelling downstream'. Treaties or conventions which define boundaries in watercourses nowadays usually refer to the thalweg as the boundary when the watercourse is navigable and nto the median line between the two banks when it is not, although it cannot be said that practice has been fully consistent". Kasikili/Sedudu Island (Bostwana/Namibia), Judgment, ICJ Reports 1999, pp. 1061 y 1062. Dissenting Opinion of Judge Weeramantry, p. 1172.

21 De esta suerte, el artículo 10 de la Convención Internacional sobre la utilización del agua se habla de necesidades humanas esenciales, de donde deriva su vinculación con los derechos humanos a nivel universal.

22 Es de inferirse que tanto la Convención Marco de las Naciones Unidas sobre Cambio Climático, de Nueva York el 9 de mayo de 1992, como su Protocolo de Kyoto de 11 de diciembre de 1997, así como el Protocolo de Montreal relativo a las Sustancias que Agotan la Capa de Ozono, de 16 de septiembre de 1987, así como las recomendaciones y principios adoptados por la Conferencia de las Naciones Unidas sobre el Medio Ambiente y el Desarrollo de 1992, en la Declaración de Río, constituyen el mínimo corpus del derecho ambiental que no podría ser ignorado por los regímenes convencionales bilaterales. 
comerciales o de inversión. ${ }^{23}$ El Tratado de Libre Comercio de América del Norte (TLCAN) es un ejemplo escolar de cómo el subdesarrollo en materia ambiental puede involucrar a Estados de muy distinta posición geopolítica y económica por razones de meras urgencias políticas o comerciales. ${ }^{24}$ Ambos Estados tampoco tienen obligaciones ambientales específicas en el marco de la Organización para la Cooperación y Desarrollo Económicos, que pudieran servir para la solución del diferendo fluvial.

\section{EL RÉGIMEN FLUVIAL ENTRE MÉXICO Y ESTADOS UNIDOS DE AMÉRICA, Y EL CAMBIO FUNDAMENTAL DE CIRCUNSTANCIAS} EN EL DERECHO INTERNACIONAL

Desde la consolidación de la noción de norma de ius cogens en los trabajos preparatorios de la Convención de Viena de 1969 sobre el Derecho de los Tratados, nada había creado mayor inestabilidad en el derecho convencional internacional, que la resurrección y sacralización del principio rebus sic stantibus en la sentencia de la Corte Internacional de Justicia en $1997 .{ }^{25}$ En rigor, la fuerza y rango que la cláusula del cambio fundamental de circunstancias ha adquirido en el derecho internacional general, supera por mucho al apoyo doctrinario y jurisprudencial que a

23 Uno de los pasajes que señala con más claridad la vocación comercial del Tribunal arbitral integrado para tal efecto señala: "El Tribunal no necesita considerar los motivos o intención para la adopción del Decreto Ecológico. De hecho, el determinar una expropiación basándose en el Decreto Ecológico, no es esencial para que el Tribunal considere que se ha violado el artículo 1110 del TLCAN. Sin embargo, el Tribunal considera que la implementación del Decreto Ecológico podría, por sí y en sí mismo, constituir un acto equivalente a una expropiación". Cfr. Metaclad Corporation v. Estados Unidos Mexicanos (caso CIADI No. ARB (AF) /97/1), Washington, D. C., 30 de agosto de 2002, p. 39.

24 De acuerdo con el artículo 104 y su Anexo 104.1 del TLCAN los tratados atinentes a la relación bilateral entre México y Estados Unidos de América son la Convención sobre el Comercio Internacional de Especies amenazadas de Flora y Fauna Silvestres, celebrada en Washington el 3 de marzo de 1973 con sus enmiendas del 22 de junio de 1979; el Protocolo de Montreal relativo a las Sustancias que Agotan la Capa de Ozono, del 16 de septiembre de 1987, con sus enmiendas del 29 de junio de 1990 cfr. Diario Oficial de la Federación, 12 de febrero de 1993, p. 3; el Convenio de Basilea sobre el Control de los Movimientos Transfronterizos de los Desechos Peligrosos y su Eliminación, del 22 de marzo de 1989; el Convenio entre los Estados Unidos Mexicanos y Estados Unidos de América sobre Cooperación para la Protección y Mejoramiento del Medio Ambiente en la Zona Fronteriza, firmado el 14 de agosto de 1983 en la Paz, Baja California Sur. Por lo que toca al Acuerdo de Cooperación Ambiental y Laboral de América del Norte, en su artículo 10, numeral 2, inciso g), se hace referencia a las cuestiones derivadas de la contaminación del aire y de los mares, sin alusión alguna a los ríos internacionales. Sin embargo, es de inferirse que los problemas fluviales bilaterales están dentro de los objetivos que se listan en el artículo 1 del referido Acuerdo.

25 Gabcíkovo-Nagymaros Project..., cit., nota 12, p. 65. 
dicha cláusula se le suele conceder en el derecho privado comparado. ${ }^{26}$ Empero la paradoja está que sea justamente en el derecho internacional general en donde un principio tan debatido haya encontrado un medio de cultivo apropiado, pues siempre se había calificado a esta rama del derecho como extraordinariamente retardataria y conservadora. La fuerza contestataria de las relaciones internacionales posteriores al fin de la guerra fría, aunada al deterioro acelerado y en algunos rubros irreversible del entorno humano, explican que fundamentalmente las circunstancias han cambiado en muchos regímenes parciales del derecho convencional internacional regional o universal, de donde se explica su revisión.

De acuerdo a las informaciones suministradas por las cancillerías de los dos Estados partes en la controversia sobre aguas fluviales, así como por la Comisión Internacional de Límites y Aguas entre México y Estados Unidos de América, ${ }^{27}$ la imposibilidad de ejecución de las condiciones pactadas en el Tratado bilateral sobre ríos internacionales no depende en absoluto de la voluntad de las partes, sino de manejo de condiciones climáticas negligentemente administradas y sometidas a un deterioro que ha terminado por dañar a las poblaciones que habitan en las dos márgenes de los ríos fronterizos. Ello surte una de las condiciones requeridas jurisprudencialmente para invocar el cambio fundamental de circunstancias, a saber, que éste debe ser imprevisible y ajeno a la actuación de las partes. ${ }^{28} \mathrm{La}$ actitud renegociadora de las partes tratando de reconducir el tratado en lo principal es revelador de la sintomatología que evoca este

26 En este mismo sentido: "De toute manière il est certain que l'application du principe du changement des circonstances ne comporte pas plus de risques d'abus que les autres causes de nullité". Cahier, Philippe, "Le Changement fondamental de circonstances et la Convention de Vienne de 1969 sur le droit des traités", Essays on honour of Roberto Ago, p. 183.

27 De esta suerte, el Acta 307 de la referida comisión (Washington, 16 de marzo de 2001), reconoce en su punto número 3: "Que los gobiernos de México y Estados Unidos de América, animados por el espíritu de amistad que prevalece en la relación entre los dos países y comprometidos a prevenir que tengan que encontrarse nuevamente en situaciones como las que aquí se han tratado, trabajen conjuntamente para identificar medidas de cooperación en materia de manejo de sequías y de manejo sustentable de esta cuenca" (énfasis añadido).

28 "A fundamental change of circumstances must have been unforeseen; the existence of the circumstances at the time of the Treaty's conclusion must have constituted an essential basis of the consent of the parties to be bound by the Treaty. The negative and conditional wording of Article 62 of the Vienna Convention on the Law of Treaties is a clear indication moreover that the stability of treaty relations requires that the plea of fundamental change of circumstances be applied only in exceptional cases". Gabcíkovo-Nagymaros Project..., cit., nota 12, p. 65. 
principio. ${ }^{29}$ pues deja de manifiesto el incumplimiento recíproco de obligaciones convencionales. ${ }^{30} \mathrm{El}$ caso del río Danubio resuelto en 1997 por la CIJ atiende en las mismas circunstancias problemas hidrológicos como los de los ríos Bravo, Colorado y Tijuana, aceptando el cambio fundamental de circunstancias y adaptando el régimen de cooperación a las nuevas relaciones internacionales para dos Estados de Europa del Este.

Otra vena de interpretación hace ya irrealizable la ejecución de las condiciones del Tratado bilateral sobre ríos internacionales. En efecto, dado el grave nivel de desertización regional y desvanecimiento paulatino de los volúmenes de irrigación (que afecta integralmente a los valles de los dos países), una excluyente de responsabilidad añejamente reconocida por el derecho internacional general, como lo es el estado de necesidad, se dibuja en las opciones jurídicas para regularizar un tratado sobre aprovechamiento fluvial que violenta las normas generales de cooperación. El estado de necesidad justifica la irrealización de obligaciones internacionales cuando se atenta a las condiciones básicas de la vida y a los factores periféricos de la supervivencia de los individuos o las comunidades sin incoar responsabilidades para éstos. El estado de necesidad apoyado por la jurisprudencia de la CIJ, ${ }^{31}$ hace concebible que un Estado como México, que demuestra carecer de volúmenes de agua apropiados para garantizar cabalmente la subsistencia de una parte importante de su población, discrimine cualquier tratado o arreglo compensatorio que pongan en riesgo sus obligaciones constitucionales y de derecho internacional sobre los derechos humanos alimentarios de sus nacionales. Por el contrario, difícilmente instancia internacional alguna podría coactivamente solicitar a México el cumplimiento de obligaciones hidrológicas (circunstancialmente ya espurias), si el incumplimiento de aquéllas tiene que ver con la racionalización de un líquido tan precioso para la vida humana y del entorno.

29 Tal es el caso del acuerdo realizado por las partes con fecha 29 de junio de 2002, por el cual se establecen suministros inferiores por parte de México, pero respetando esencialmente el tratado bilateral sobre ríos internacionales.

30 "The Court would set a precedent with disturbing implications for treaty relations and the integrity of the rule pacta sunt servanda if it were to conclude that a treaty in force between States, which the parties have implemented in considerable measure and at great cost over a period of years, might be unilaterally set aside on grounds of reciprocal noncompliance". Gabcíkovo-GabcíkovoNagymaros Project..., cit., nota 12, p. 68.

31 “...Even if found justified, it does not terminate a Treaty; the Treaty may be ineffective as long as the condition of necessity continues to exist; it may in fact be dormant, but —unless the parties by mutual agreement terminate the Treaty - it continues to exist. As soon as the state of necessity ceases to exist, the duty to comply with treaty obligations revives". Gabcíkovo-Nagymaros Project..., cit., nota 12, p. 63. 


\section{El PolíGONO OCCIDENTAL EN EL GOLFO DE MÉXICO Y EL TIMOR GAP}

\section{Gaps en el derecho internacional del mar}

El esfuerzo codificador del derecho del mar y la tarea paralela de la Corte Internacional de Justicia en materia de resolución de controversias en el ámbito de delimitación de la plataforma continental o de fronteras marítimas, se han desarrollado bajo el influjo de una necesidad apremiante de solucionar estos diferendos, sin existir un derecho consuetudinario de base lo suficientemente explícito para servir de derecho sustantivo y con una endeble lege ferenda representada por las diversas y erráticas convenciones multilaterales en materia de derecho del mar, cuyo carácter ecléctico las hizo siempre inútiles para regir las relaciones marítimas de la comunidad internacional.

Es ya tópico narrar en los ejercicios académicos todas las anécdotas que caracterizaron el desarrollo del derecho del mar en la postguerra. Interesante pero ya prácticamente inútil, es describir los primeros actos unilaterales de los Estados reclamando las adyacencias territoriales de sus espacios continentales derivados de la celebérrima Proclama Truman; el proceso de consolidación de la Convención de Ginebra sobre la Plataforma Continental, ${ }^{32}$ la cual en 1969 ya estaría en crisis; $; 3$ así como la convocatoria y firma de la Tercera Convención de las Naciones Unidas sobre

32 La doctrina jurídica recibió con gran entusiasmo la elaboración de las cuatro convenciones derivadas de la Conferencia de 1958, cfr. Gómez Robledo, Antonio, "La Convención de Ginebra sobre la Plataforma Continental", Revista de la Facultad de Derecho de la Universidad Nacional Autónoma de México, t. VIII, abril-junio de 1958, núm. 30, p. 7. Es importante destacar que el artículo 6 de la Convención de Ginebra sobre delimitación de Plataforma Continental de 1958 tiene una lógica que fue incomprendida por la doctrina y jurisprudencia internacionales. En efecto, los criterios de delimitación ahí contenidos, son completamente conformes con los principios y necesidades de delimitación del derecho interamericano, mismos que son acordes con la proclama Truman. La configuración particular del Continente Americano, que es el único en el planeta que corre de polo a polo y en donde la configuración política es la de Estados gigantescos con fachadas marítimas orientadas en la misma dirección siempre hacia el mar abierto, hace comprensible tanto la mencionada proclama como el referido artículo 6, pues en nuestro continente la idea de Estados que se hiciesen frente es prácticamente ajena a la estructura geomorfológica y política del continente. En último de los rigores, la pretensión de la corte de aplicar un principio del derecho interamericano, a la delimitación de los espacios marítimos en el Mar del Norte, fue un acto extralógico de efectos evidentemente nocivos para la jurisprudencia ulterior en la materia.

33 Cfr. North Sea..., cit., nota 11, p. 51, para. 96. 
el Derecho del Mar de 1982, conocida como Montego Bay, la cual fue una crisis en su elaboración y otra crisis en su entrada en vigor en 1994. ${ }^{34}$

Es también muy conocido que la jurisprudencia de la $\mathrm{CIJ}^{35}$ tuvo que hacerse a la labor de crear un derecho pretoriano en materia del mar, sin derecho consuetudinario o convencional alguno como ya se dijo, con la incapacidad lógica de apelar a los principios generales de derecho, pues el derecho interno de los Estados no delimita espacios marítimos internacionales, hasta el punto en que casi sólo con la Ética Nicomaquea de Aristóteles en la mano, la Corte de La Haya tuvo que hacer todo el trabajo, creando una compleja e inagotable doctrina sobre los principios equitativos (como método y como fin) de la delimitación marítima, aparejados de innumerables criterios o factores que se sumaron a recurrentes circunstancias relevantes o pertinentes que en último de los rigores poco incidieron en la identificación geológica de las plataformas continentales y en su adscripción a los Estados ribereños. En el momento actual se califica como apostasía la puesta en duda de los criterios de delimitación de la plataforma continental, no obstante que en ocasiones en las sentencias más sesudas de la corte se confundió a ésta con la frontera marítima, y en ocasiones fue irrelevante la inexistencia de una plataforma para que se aplicase la misma metodología de delimitación. ${ }^{36}$

Estas experiencias hicieron crecer infinitamente a la Corte Internacional de Justicia, y no obstante nuestra crítica, podemos reconocer que ahí estuvo el verdadero desarrollo del derecho del mar en el último cuarto del siglo veinte. Sin embargo, la suerte fue amarga para la corte en dos casos de vocación marítima de distintas latitudes, mismos que habrían de mos-

34 Cfr. "Law of the Sea Forum: The 1994 Agreement on Implementation of the Seabed Provisions of the Convention of the Law of the Sea", American Journal of International Law, vol. 88, núm. 4, octubre de 1994: "The Entry into Force of the Convention on the Law of the Sea: A Redistribution of Competences between States and International Organisations in Relation to the Management of the International Commons?", Zeitschrift für ausländisches öffentliches Recht und Völkerrecht, Heidelberg, Verlag W. Kohlhammer, 55/2, 26-28 de enero de 1995. En sentido contrario, véase Gómez-Robledo Verduzco, Alonso, "Acuerdo vigente desde el 28 de julio de 1996, relativo a la aplicación de la parte XI de la Convención de las Naciones Unidas sobre el Derecho del Mar", Boletín Mexicano de Derecho Comparado, núm. 89, 1997.

35 Cfr. North Sea..., cit., nota 11; Continental Shelf (Tunisia/Libyan Arab Jamahiriya), Judgment, ICJ Reports 1982; Delimitation of the Maritime Boundary in the Gulf of Maine Area, Judgment, ICJ Reports 1984; Continental Shelf (Libyan Arab Jamahiriya/Malta), ICJ Reports 1985; Land, Island..., cit., nota 19; Maritime Delimitation in the Area between Greenland and Jan Mayen, Judgment, ICJ Reports 1993. De alguna manera: East Timor (Portugal v. Australia), Judgment, ICJ Reports 1995.

36 Cfr. Delimitation of the Maritime..., cit., nota anterior, p. 315; Maritime Delimitation..., cit., nota anterior, p. 59. 
trar los vacíos y la oblicuidad de la evolución del derecho del mar. En el primero de ellos, que opuso a El Salvador contra Honduras en 1992, ${ }^{37}$ en donde la corte (en sala) tuvo que reconocer una excepción al principio de apertura y libertad de los mares en detrimento de la comunidad internacional y a favor de El Salvador, Honduras y Nicaragua, ${ }^{38}$ lo que hizo de la corte un blanco ineludible de severas críticas técnicas. ${ }^{39}$ Posiblemente la inestabilidad ulterior de la sentencia derivó de que fue sólo una sala quien dictó la resolución y no el pleno de la corte.

La historia se repetiría para malestar del Tribunal de La Haya años después. La controversia que opuso a Portugal contra Australia respecto de la delimitación de la plataforma continental entre este último y Timor Oriental fue un caso aleccionador en más de un sentido. ${ }^{40}$ Los espacios marítimos sólo se estudiaron de paso dados los problemas competenciales que ahí se dilucidaron. Empero, la corte y particularmente los jueces en sus opiniones individuales y disidentes, dibujaron la problemática del llamado Timor Gap, esto es una parte no delimitada de la plataforma continental que las agencias australiana e indonesia habían preferido no tocar en espera de mejores tiempos (tal y como ocurrió entre México y Estados Unidos de América en la zona del Golfo). Ese vacío geológico (y diplomático) ha sido históricamente revelador, pues se trata de una mezcla de desidia, falta de titularidad de derechos y amedrentamiento recíproco lo que llevó a los dos Estados a mantener bajo un régimen de indefinición las plataformas continentales respecto de las zonas donde ambas se sobreponen, que es aproximadamente a la mitad del así llamado Timor Gap. ${ }^{41}$ De alguna manera, los dos casos anteriores ilustran los vacíos técnicos que tanto la Convención de Ginebra de 1958 así como la Convención de las Naciones Unidas de 1982 sobre el Derecho del Mar dejaron. Vacíos

37 Land, Island..., cit., nota 19.

38 La encrucijada de derecho internacional del mar (regional) fue discernida por la corte en los siguientes términos: "To have recognized exclusive 'maritime belts' along the litoral inside those 'territorial waters', the property of the three States in common, was no doubt an anomaly in terms of the modern law of the sea; but it was in accord with what had emerged from actual practice of the coastal States in the Gulf of Fonseca at that time..." (énfasis en el original). Land, Island..., cit., nota 19, p. 592. Para una idea similar $c f r$. The Minquiers and Ecrehos case, Judgment of November 17th, 1953, ICJ Reports 1953, p. 52.

39 "In sum, the concept of 'historic waters' has become practically a redundancy...", Land, Island..., cit., nota 19, p. 756.

40 Cfr. East Timor... cit., nota 35, p. 98.

41 Cfr.ibidem, p. 110. 
claro, que se permitieron por la connivencia de los Estados involucrados y que habría de tener consecuencias en el derecho internacional general.

Sin embargo, existe una diferencia medular entre los tres asuntos. Mientras los casos del Golfo de Fonseca y de Timor Oriental son asuntos contenciosos, la delimitación de la plataforma continental en la zona del Golfo de México es convencional, esto es el resultado de un tratado bilateral que pone fin a cualquier consideración de equidad o de proporcionalidad. Así, por desafortunado que sea un acuerdo final, toda la jurisprudencia internacional en materia de delimitación de plataforma continental o de frontera marítima es simplemente inaplicable, quedando sujeto al principio contractual de la autonomía de la voluntad de las partes ${ }^{42}$ como lo reconociera la propia Corte Internacional de Justicia. ${ }^{43}$

\section{Gaps en la relación bilateral}

La historia de un Estado nacional puede ser diagnosticada por la suscripción de sus tratados fronterizos. La firma de un tratado internacional en materia de límites (territoriales o marítimos, da igual), invoca de inmediato a todos los protagonistas de la política interna, al tiempo de que pone a la diplomacia del referido Estado en su punto específico de equilibrio en la relación bilateral o en el contexto regional de que se trate. La aprobación siempre diferida de los tratados de límites marítimos en la zona del Golfo de México, era cronológicamente inevitable. ${ }^{44}$

El caso de la delimitación de la plataforma continental entre México y Estados Unidos de América en la zona del Golfo parece presentar la

42 "The Court's conclusion that the Treaty contains an agreed boundary renders it unnecesary to consider the history of the "Borderlands..."'. The Territorial Dispute (Libyan Arab Jamahiriya $v$. Chad), Judgment of 3 February 1994, ICJ Reports 1994, p. 38.

43 "But in the context of relations governed by treaties, it is always for the parties concerned to decide, by agreement, in what conditions their mutual relations can best be balanced". Maritime Delimitation in the Area between Greenland and Jan Mayen, Judgment, ICJ Reports 1993, p. 77.

44 Cfr. Decreto Promulgatorio del Tratado entre el gobierno de los Estados Unidos Mexicanos y el gobierno de Estados Unidos de América sobre la delimitación de la Plataforma Continental en la Región Occidental del Golfo de México más allá de la doscientas millas náuticas, Diario Oficial de la Federación, 22 de marzo de 2001, p. 8. Decreto de aprobación, Diario Oficial de la Federación, 16 de enero de 2001, p. 2. El decreto senatorial de aprobación se realizó con una "corrección" de suyo reveladora, negociada por las partes a última hora, respecto del párrafo 1, inciso a) del artículo $\mathrm{V}$ del tratado, al siguiente tenor: "A medida que se vaya generando la información geológica y geofísica que permita facilitar el conocimiento de las Partes sobre la posible existencia de yacimientos transfronterizos, incluyendo las notificaciones de las Partes de acuerdo con el párarfo 6 del Artículo IV, las Partes se reunirán periódicamente con el fin de identificar, localizar y determinar las características geológicas y geofísicas de dichos yacimientos”. Cfr. Diario Oficial de la Federación, 16 de enero de 2001, p. 2. 
misma sintomatología que el Timor Gap: un abandono político bilateral evidente, una ausencia de información técnica total, una premura superveniente para resolver el conflicto y una diplomacia críptica que marginó a los sectores interesados en coadyuvar a la solución del asunto. ${ }^{45} \mathrm{La}$ citada delimitación ha sido asunto de muy pocos, por lo que concierne a la parte mexicana.

Las características de la plataforma continental en la zona del Golfo de México es la de un continuum geológico ${ }^{46}$ con una línea isóbata regular en prácticamente todos sus puntos, no obstante sus proporciones gigantescas. Su propia conformación da cuenta de cuatro horizontes geológicos generadores de hidrocarburos situados en las eras del Jurásico, Cretácico, Eoceno y Mioceno. Dichos horizontes fueron reconocidos en la propia área del Polígono Occidental por debajo del lecho marino en el rango de 4,000 y 8,000 metros de profundidad. ${ }^{47}$ Se trata de una superficie en forma de planicie sin grandes estructuras que se traslapen entre sí como ha ocurrido en otras latitudes y que ha dado a tales controversias ante la CIJ un cariz fundamentalmente geomorfológico. Las descomunales riquezas petrolíferas y de gas natural que ahí se albergan parecen estar fuera de dudas para ambos Estados. Sin embargo tales recursos presentan dificultades de exploración y explotación a 10,000 pies de profundidad. ${ }^{48}$ El caso de México y Estados Unidos de América en la zona del Golfo es que la configuración natural del espacio marítimo hace que ambos Estados sean al propio tiempo adyacentes en un punto y que se hagan frente en otros.

El tratado que ha dividido la plataforma continental entre ambos países es rigurosamente equidistante, deja intacta la columna superior de las aguas, estableciendo únicamente un régimen de escisión para la platafor$\mathrm{ma},{ }^{49} \mathrm{y}$ se ha hecho bajo un confeso desconocimiento de la existencia de

45 Ello ha sido a tal grado secreto, que ante la negativa de la autoridad de proporcionar información elemental sobre el proceso de negociación de la plataforma mexicana, fue incluso necesario el entablamiento de un juicio de garantías por violación al artículo 6 de la Carta Federal de México (derecho de información), para intentar obtenerla, $c f r$. Juan de Dios Gutiérrez Baylón $v$. Secretario de Relaciones Exteriores, juicio de amparo P.-668/2000.

$46 C f r$. Vargas, Jorge, "El régimen legal de México sobre sus espacios marinos: una propuesta para la delimitación de la plataforma continental en la parte más profunda del Golfo de México", Revista de Investigaciones Jurídicas, México, Escuela Libre de Derecho, núm. 21, 1997, p. 722.

47 Cfr. Limón González, Mario, "Proyecto Polígono Occidental”, Anuario Mexicano de Derecho Internacional, vol. II, UNAM, 2002, p. 103.

48 Cfr. Vargas, Jorge, "El régimen legal de México sobre sus espacios marinos", p. 725.

49 Cfr. "U.S. Mexico Continental Shelf Boundary in Gulf of Mexico", American Journal of International Law, vol. 95, núm. 2, 2001, pp. 393 y 394. 
ciertos yacimientos petrolíferos en la zona limítrofe, por lo que se creó una efímera demarcación restringida (gap) denominada "El Área", ${ }^{50}$ en donde los Estados no realizarán perforación o explotación petrolera o de gas alguna, lo que es de suyo revelador de las condiciones de negociación. Es fácilmente inferible que así como se desconocen los depósitos en la línea de delimitación también se ignore por completo la existencia de cualesquiera otros reductos energéticos en el resto de la zona de repartición, diversos a "El Área", por lo que no es posible saber qué recursos perdimos por negociar a ciegas, no obstante que era eventualmente defendible el argumento de una prolongación natural de nuestra plataforma continental, ${ }^{51}$ argumento éste que nunca pudo ser cabalmente estructurado por los sectores académicos y científicos nacionales, dado el ya señalado celo bonapartista con el cual la cancillería mexicana decidió manejarlo a su exclusivo riesgo. Seguramente este asunto ocupará capítulos especiales en los libros de historia nacional del futuro.

La formación de este nuevo gap interamericano es un típico diferimiento diplomático a un problema en todos sentidos profundo. El plazo de diez años se verá rápidamente vencido sin que nuestro desarrollo tecnológico o nuestra relación bilateral con Estados Unidos de América nos permita tener más información sobre los posibles yacimientos transfronterizos. De la lectura del tratado se deduce que al menos los mexicanos no sabemos lo que existe en el subsuelo de la plataforma continental en "El Área", lo que nos pone en una situación espeluznantemente desventajosa para negociar con aquel que tiene la información sobre el objeto del contrato, además del objeto mismo. De hecho se ha afirmado que las empresas petroleras americanas hace ya tiempo que cuentan con los conoci-

50 "El Área" es un espacio de 1.4 millas naúticas de cada lado de la línea de delimitación (lo que en total le da un espesor de 2.8 millas náuticas), en donde se hace una proscripción temporal de explotación petrolera o de gas natural en la plataforma continental, durante diez años a partir de la entrada en vigor del tratado.

51 Ciertamente dicho argumento no habría estado exento de complejidad, ya que como se ha descrito líneas arriba, pudiera ser que la zona de delimitación sea absolutamente homogénea y no pudiera encontrarse una prolongacion natural de una posible plataforma puramente mexicana que al propio tiempo se proyectara sobre la estadounidense. Empero, no obstante que en el mejor de los casos se hubiese llegado al mismo resultado que el derivado del tratado, resulta inadmisible que los ciudadanos de un Estado nos encontremos abiertamente marginados de la negociación de las fronteras nacionales. 
mientos (y los métodos) para acceder a tales profundidades con fines de explotación. ${ }^{52}$

La historia bilateral no pasa en vano y desde estos momentos debemos ya estar profundamente preocupados sobre el futuro régimen petrolero de "El Área" ${ }^{53}$ Es de reconocerse que se requiere una buena dosis de ingenuidad para negociar en estos términos un tratado que a la vez que fronterizo es particularmente importante en materia de recursos energéticos. No podemos voltear la mirada hacia el pasado y criticar a nuestros antecesores por hacer malos tratos territoriales con los americanos, si nosotros hemos sido capaces de aprobar un tratado en donde se pactó que va a ser Estados Unidos de América quien nos van a ir "facilitando" ${ }^{54}$ el conocimiento que ellos recaben sobre la existencia de la riqueza petrolera, para que entonces los mexicanos podamos negociar con un mayor conocimiento de causa en defensa de nuestros intereses nacionales.

\section{CONCLUSIONES}

En los diferendos fluviales y marítimos de México y Estados Unidos de América se entreveran problemas técnicos, secuelas del subdesarrollo y enfrentamientos de la dialéctica centro y periferia, que si bien pudiera no coincidir con los usos del lenguaje académico en boga, sigue describiendo un mecanismo de dependencia que incide en las expectativas de bienestar para los habitantes de una región del mundo de gran importancia poblacional como es México.

La incapacidad de negociación y revisión de tratados fluviales o marítimos más ventajosos para el desarrollo nacional por efecto de la verticalidad diplomática, puede ser abatida con una incorporación más decidida de los problemas de esta naturaleza a principios universales consagrados por el derecho internacional general. Dicha labor de gran complejidad jurídica y política, tiene como punto de partida la autoafirmación de los principios y criterios sustentados por la jurisprudencia y doctrina internacionales (incluyendo un mayor apoyo en la doctrina nacional ati-

52 Cfr. García Moreno, Víctor Carlos, "El problema de las 'donas' del Golfo de México; enfoque jurídico-internacional”, Revista Lex, núms. 36-37, junio-julio de 1998, p. 79.

53 El artículo $\mathrm{V}$, numeral 1, inciso b), contiene la promesa de que tras la entrada en vigor del tratado, las partes “...buscarán llegar a un acuerdo para la eficiente y equitativa explotación de dichos recursos transfronterizos...”.

54 Cfr. artículo V, numeral 1, inciso a, del tratado. 
nente). Sería un lugar común postular la necesidad de revisar por completo los tratados estudiados en este artículo, asimismo rebasa a una seria posición cientista, imaginar que tales convenciones internacionales fueron manipuladas de manera inconfesa por nuestros cosignatarios con el fin de inferirnos males prefabricados. Todo ello es falso. Sin embargo, es importante que un bagaje jurídico internacional más amplio apoye las negociaciones bilaterales sobre delimitación marítima o aprovechamientos hidrológicos fluviales, preparando el terreno para eventuales contenciosos internacionales.

Estimamos que el cambio fundamental de circunstancias o la excluyente de responsabilidad del estado de necesidad, enarbolados por la reciente jurisprudencia internacional, abre el paso ya sea a una revisión del tratado sobre ríos internacionales o de su legítima inejecución. No sostenemos que al acogerse a tales fórmulas, la unilateralidad por parte de México sea una solución aceptable para los problemas fluviales fronterizos, sino bien por el contrario, que es imperativo resolverlos en foros multilaterales, en aplicación del derecho internacional general.

Por lo que toca al Polígono Occidental en el Golfo de México, tanto los criterios de delimitación como sus resultados afectan el régimen fronterizo de México, siendo de muy alta probabilidad que en aplicación del derecho internacional general, la prolongación natural de nuestro territorio nos hubiese llevado a una adjudicación más satisfactoria.

\section{BIBLIOGRAFÍA}

\section{Libros}

BoGDANOVIC, Slavko (coord.), International Law of Water Resources, The Netherlands, Kluwer Law International, 2001.

Brownlie, Ian, Principles of Public International Law, Oxford, Fifth Edition, 1998.

ENRÍQUEZ COYRO, Ernesto, El tratado entre México y Estados Unidos de América sobre ríos internacionales, México, UNAM, 1976, t. II.

KISS, Alexandre, Droit international de l'environnement, París, Pedone, 1989.

NGUYEN, Quoch Dinh et al., Droit international public, LGDJ, 1994. TORRES, Blanca, México y el mundo. Historia de sus relaciones exteriores, t. VIII. 
ZorAIDA VÁZqueZ, Josefina y MEYer, Lorenzo, México frente a Estados Unidos, México, Fondo de Cultura Económica, 1994.

ZorriLla, Luis G., Historia de las relaciones entre México y Estados Unidos de América, México, Porrúa, 1977, t. II.

\section{Artículos}

BENVENISTI, Eyal, "Collective Action in the Utilization of Shared Freshwater: The Challenges of International Water Resources Law", American Journal of International Law, vol. 90, núm. 3, julio de 1996. BRunNÉE, Jutta y ToOPE, Stephen J., "The Changing Nile Basin Regime: Does Law Matter?", Harvard International Law Journal, vol. 43, núm. I, 2002.

CAHIER, Philippe, "Le Changement fondamental de circonstances et la Convention de Vienne de 1969 sur le droit des traités", Essays on honour of Roberto Ago.

ESQUIVEL OBREGÓN, Toribio, "Impugnación del Tratado de Aguas", Revista de la Escuela Nacional de Jurisprudencia, México, UNAM, t. VIII, núm. 30, 1946.

GARCía Moreno, Víctor Carlos, "El problema de las 'donas' del Golfo de México; enfoque jurídico-internacional”, Revista Lex, núms. 3637, junio-julio de 1998.

GómEZ RoBledo, Antonio, "La Convención de Ginebra sobre la Plataforma Continental", Revista de la Facultad de Derecho de la Universidad Nacional Autónoma de México, t. VIII, núm. 30, abril-junio de 1958.

GÓMEZ-ROBLEDo VERDUZCO, Alonso, “Acuerdo vigente desde el 28 de julio de 1996, relativo a la aplicación de la parte XI de la Convención de las Naciones Unidas sobre el Derecho del Mar", Boletín Mexicano de Derecho Comparado, núm. 89, 1997.

"Law of the Sea Forum: The 1994 Agreement on Implementation of the Seabed Provisions of the Convention of the Law of the Sea", American Journal of International Law, vol. 88, núm. 4, octubre de 1994.

LIMÓN GONZÁLEZ, Mario, "Proyecto Polígono Occidental", Anuario Mexicano de Derecho Internacional, México, UNAM, vol. II, 2002. MARGADANT S., Guillermo F., "El régimen jurídico de las aguas no marítimas en la literatura iusromanista desde los posglosadores hasta 
mediados del siglo XVIII", Boletín Mexicano de Derecho Comparado, México, UNAM, 1986.

"The Entry into Force of the Convention on the Law of the Sea: A Redistribution of Competences between States and International Organisations in Relation to the Management of the International Commons?", Zeitschrift für ausländisches öffentliches Recht und Völkerrecht, Heidelberg, Verlag W. Kohlhammer, 55/2, 26-28 de enero de 1995.

"U. S. Mexico Continental Shelf Boundary in Gulf of Mexico", American Journal of International Law, vol. 95, núm. 2, 2001.

VARGAS, Jorge, "El régimen legal de México sobre sus espacios marinos: una propuesta para la delimitación de la plataforma continental en la parte más profunda del Golfo de México", Revista de Investigaciones Jurídicas, México, Escuela Libre de Derecho, núm. 21, 1997.

\section{Jurisprudencia internacional}

\section{A. Casos arbitrales}

Metaclad Corporation v. Estados Unidos Mexicanos, (caso CIADI No. ARB (AF) /97/1), Washington, D. C., 30 de agosto de 2002.

The Trail Smelter case (United States of America v. Canada), Award of March 11, 1941, Reports of International Arbitral Awards, United Nations, vol. III.

\section{B. Casos ante la Corte de Justicia Centroamericana}

Golfo de Fonseca, sentencia, párrafo III, 9 de marzo de 1917.

\section{Casos ante la Corte Internacional de Justicia}

Continental Shelf (Libyan Arab Jamahiriya/Malta), ICJ Reports 1985.

Continental Shelf (Tunisia/Libyan Arab Jamahiriya), Judgment, ICJ Reports 1982.

Delimitation of the Maritime Boundary in the Gulf of Maine Area, Judgment, ICJ Reports 1984.

East Timor (Portugal v. Australia), Judgment, ICJ Reports 1995.

Fisheries case (United Kingdom $v$. Norway), Judgment of December 18 th, 1951, ICJ Reports 1951. 
Fisheries Jurisdiction (Spain $v$. Canada), Jurisdiction of the Court, Judgment, ICJ Reports 1998.

Gabcíkovo-Nagymaros Project (Hungary/Slovakia), Judgment, ICJ Reports 1997.

Kasikili/Sedudu Island (Bostwana/Namibia), Judgment, ICJ Reports 1999.

Land, Island and Maritime Frontier Dispute (El Salvador/Honduras: Nicaragua interveining), Judgment of 11 September 1992, ICJ Reports 1992.

Legality of the Threat or Use of Nuclear Weapons, Advisory Opinion, ICJ Reports 1996.

Maritime Delimitation in the Area between Greenland and Jan Mayen, Judgment, ICJ Reports 1993.

North Sea Continental Shelf, Judgment, ICJ Reports 1969.

The Corfu Channel, Judgment, Dissenting Opinon by Judge Azevedo, ICJ Reports 1949.

The Minquiers and Ecrehos case, Judgment of November 17th, 1953, ICJ Reports 1953.

The Territorial Dispute (Libyan Arab Jamahiriya v. Chad), Judgment of 3 February 1994, ICJ Reports 1994.

\section{Documentos}

Acta 307, Comisión Internacional de Límites y Aguas entre México y Estados Unidos de América, Washington, 16 de marzo de 2001.

\section{Tratados}

Convención para el Establecimiento de una Comisión Internacional que decida las Cuestiones de Límites que se susciten en el Cauce de los Ríos Bravo del Norte y Colorado, firmado en la ciudad de Washington el 1 de marzo de 1889.

Convención Marco de las Naciones Unidas sobre Cambio Climático, Nueva York, 9 de mayo de 1992.

Convention sur le droit relatif aux utilisations des cours d'eau internationaux à des fins autres que la navigation, Rapport de la Sixième Commission réunie en Groupe de travail plénier, Président M. Chusei Yamada, A/51/869, 1997. 
Protocolo de Kyoto de la Convención Marco de las Naciones Unidas sobre Cambio Climático, 11 de diciembre de 1997.

Protocolo de Montreal relativo a las Sustancias que Agotan la Capa de Ozono, 16 de septiembre de 1987,

Tratado relativo al Aprovechamiento de las Aguas de los Ríos Colorado y Tijuana, y del Río Bravo (Grande) desde Fort Quitman, Texas, hasta el Golfo de México. Firmado en Washington, D. C. el 3 de febrero de 1944.

Tratado entre el gobierno de los Estados Unidos Mexicanos y el gobierno de Estados Unidos de América sobre la Delimitación de la Plataforma Continental en la Región Occidental del Golfo de México más allá de la Doscientas Millas náuticas, Diario Oficial de la Federación, 22 de marzo de 2001. 